\title{
Small molecule photocatalysis enables drug target identifi- cation via energy transfer
}

Aaron D. Trowbridge ${ }^{1 *}$, Ciaran P. Seath ${ }^{1 *}$, Frances P. Rodriguez-Rivera ${ }^{2 * \dagger}$, Beryl X. Li $^{1}$, Barbara E. Dul ${ }^{3}$, Adam G. Schwaid ${ }^{4}$, Jacob B. Geri ${ }^{1}$, James V. Oakley ${ }^{1}$, Olugbeminiyi O. Fadeyi ${ }^{5}$, Rob C. Oslund ${ }^{5}$, Keun Ah Ryu ${ }^{5}$, Cory White $^{5}$, Tamara Reyes-Robles ${ }^{5}$, Paul Tawa ${ }^{6}$, Dann L. Parker, Jr. ${ }^{2 \dagger}$, David W. C. MacMillan ${ }^{1 \dagger}$

\section{Abstract:}

The identification of cellular targets that can be exploited for therapeutic benefit, broadly known as target ID, remains a fundamental goal in drug discovery. In recent years, the application of new chemical and biological technologies that accelerate target ID has become commonplace within drug discovery programs, as a complete understanding of how molecules react in a cellular environment can lead to increased binding selectivity, improved safety profiles, and clinical efficacy. Established approaches using photoaffinity labelling (PAL) are often costly and timeconsuming due to poor signal-to-noise coupled with extensive probe optimization. Such challenges are exacerbated when dealing with low abundance membrane proteins or multiple protein target engagement, typically rendering target ID unfeasible. Herein, we describe a general platform for photocatalytic small molecule target ID, which hinges upon the generation of high-energy carbene intermediates via visible light-mediated Dexter energy transfer. By decoupling the reactive warhead from the drug, catalytic signal amplification results in multiple labelling events per drug, leading to unprecedented levels of target enrichment. Through the development of cell permeable photocatalyst conjugates, this method has enabled the quantitative target and off target identification of several drugs including (+)-JQ1, paclitaxel, and dasatinib. Moreover, this methodology has led to the target ID of two GPCRs ADORA2A and GPR40 - a class of drug target seldom successfully uncovered in small molecule PAL campaigns.

\section{Main text:}

The identification of biological targets and understanding of their interactions at the molecular level (target ID) is essential for the successful design of new therapeutic candidates and their progression into the clinic ${ }^{1,2}$. In recent years however, the intrinsic challenges associated with fully characterizing drug targets has manifested in low success rates and lengthy timelines, resulting in an industry-wide bottleneck within the developmental pipeline ${ }^{3,4}$. Therefore, the development of new methods to elucidate small molecule targets has the potential to significantly increase the success of therapeutic target selections, which should in turn lead to a reduction in clinical attrition and ultimately patient morbidity (Scheme 1a) ${ }^{1,5,6}$.

Over the last two decades, technological advancements in the fields of mass spectrometry ${ }^{7}$, chemical genetics $^{8}$, and bioinformatics ${ }^{9}$ have transformed drug target identification leading to improvements in our understanding of biological pathways and cellular signalling ${ }^{2,10}$. However, while this information has provided a more focused route to the complex process of drug discovery, there remains a demand for target identification technologies for proteins without a well-described mechanism-of-action ${ }^{11}$. To address this need, affinity-based approaches ${ }^{12}$, and photoaffinity labelling (PAL) in particular, have now become routinely used tools in drug discovery (Scheme 1a) ${ }^{13}$. PAL works by the incorporation of a stoichiometric photoactivatable group, such as a diazirine, and an affinity handle, such as biotin, into the small-molecule architecture ${ }^{14}$. Following UV-activation and affinity-based enrichment, immunoblotting and proteomic analysis can be used to gather information regarding the identity of the target $\operatorname{protein}^{15}$. 
bioRxiv preprint doi: https://doi.org/10.1101/2021.08.02.454797; this version posted August 2, 2021. The copyright holder for this preprint (which was not certified by peer review) is the author/funder, who has granted bioRxiv a license to display the preprint in perpetuity. It is made available under aCC-BY-NC-ND 4.0 International license.

a. Small molecule target ID in phenotypic screeing-based drug discovery

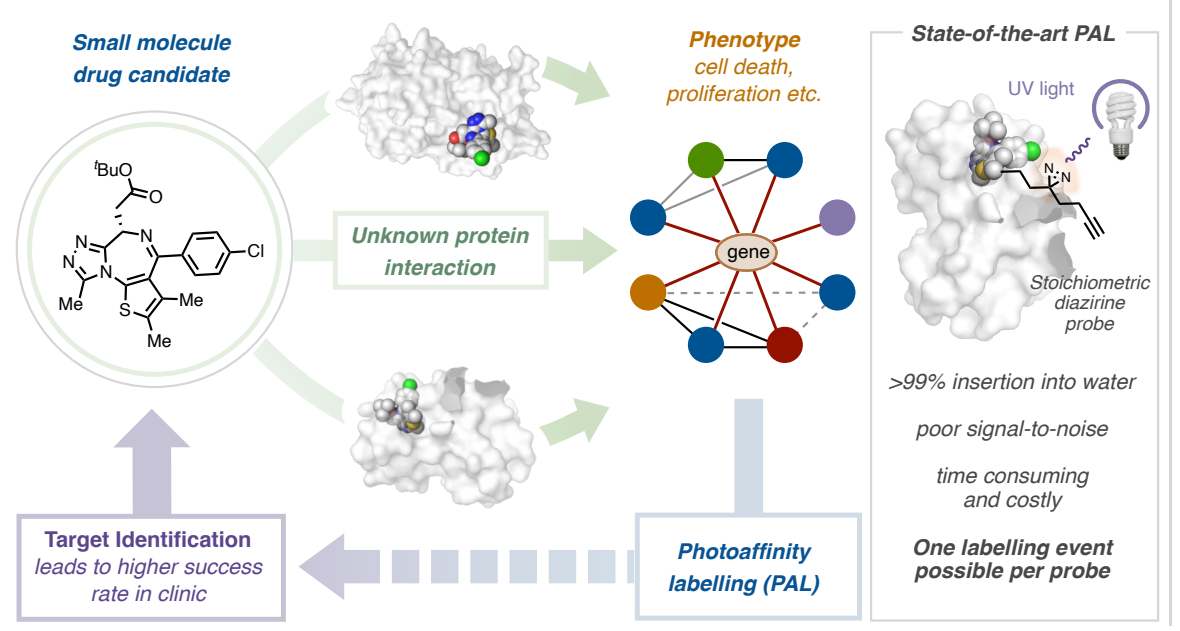

c. Development of intracellular $\mu$ Map catalyst suitable for small molecule target ID

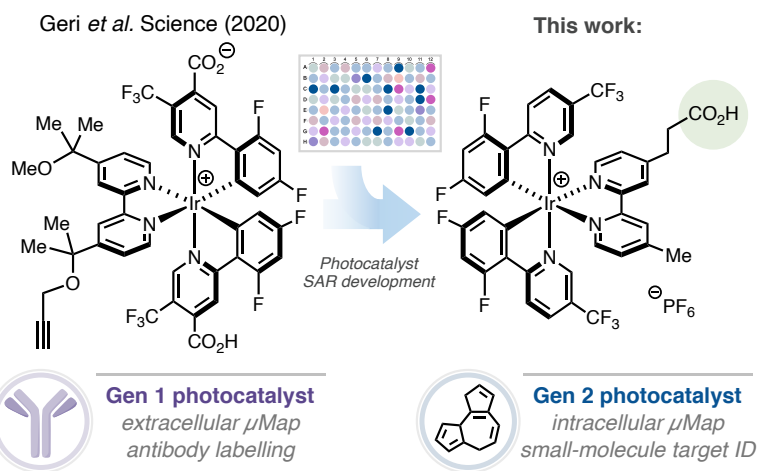

\section{Carboxylic acid conjugation handle \\ Cell permeable \\ Commercially available}

No SAR required

Multiple derivatives prepared (Ir-PEG3$\mathrm{NH}_{2}, \mathrm{Ir}-\mathrm{DBCO}, \mathrm{Ir}-$ PEG3- $\mathrm{CO}_{2} \mathrm{H}$ ) b. Photocatalytic target ID enabled by $\mu$ Mapping
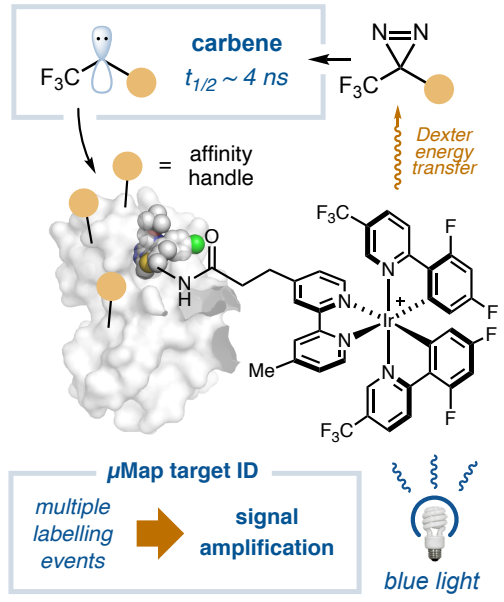

d. $\mu$ Map photocatalyst cell permeability

Scheme 1. Photoaffinity labelling comprises a critical component of small molecule target ID. a, Target ID campaigns are critical for the development of successful drugs, though often rely on challenging photoaffinity labelling campaigns that employ the stoichiometric activation of diazirine smallmolecule conjugates with UV light. b, Our approach separates the warhead from the small molecule probe, instead employing the photocatalytic activation of diazirines using visible light, giving rise to significant signal enhancement. c, Development of cell-penetrating, generation 2 photocatalyst suitable for small-molecule conjugation and target ID. d, Cell permeability of Ir-photocatalysts determined by halotag chaser assay; photocatalyst PEG-hexyl chloride conjugate and TAMRA hexyl chloride incubated with HEK293T cells expressing TOM20-halotag. Western blot analysis and immunostaining TOM20 with anti-TAMRA reveals off-compete only in the presence of Ir-G2 catalyst.

While these methods have been extremely empowering for a number of protein classes ${ }^{16-18}$, practically they remain capricious and typically struggle to determine the complete interactome due to low receptor and protein abundance and short half-lives, leading to low cross-linking yields and high background ${ }^{15,19}$. The use of diazirinebased probes in particular has been challenging in this context as $>99 \%$ of the carbenes generated upon UV irradiation react with water and not the target ${ }^{20}$. These spent probes serve to further block the binding of unreacted molecules, further hampering labelling efficiency. As a result, costly and time-consuming structural optimization campaigns are often required to overcome these shortfalls.

Indeed, the inherent difficulties associated with PAL have inspired the development of several elegant methods that hinge upon the use of stoichiometric activated electrophiles ${ }^{12,21-24}$, single-electron transfer events ${ }^{25}$, or specific oxidizable residues ${ }^{26}$ to identify target proteins. However, many of these technologies are limited to a single labelling event per drug molecule, often require extensive structural optimization (linker length and composition), and 
bioRxiv preprint doi: https://doi.org/10.1101/2021.08.02.454797; this version posted August 2, 2021. The copyright holder for this preprint (which was not certified by peer review) is the author/funder, who has granted bioRxiv a license to display the preprint in perpetuity. It is made available under aCC-BY-NC-ND 4.0 International license.
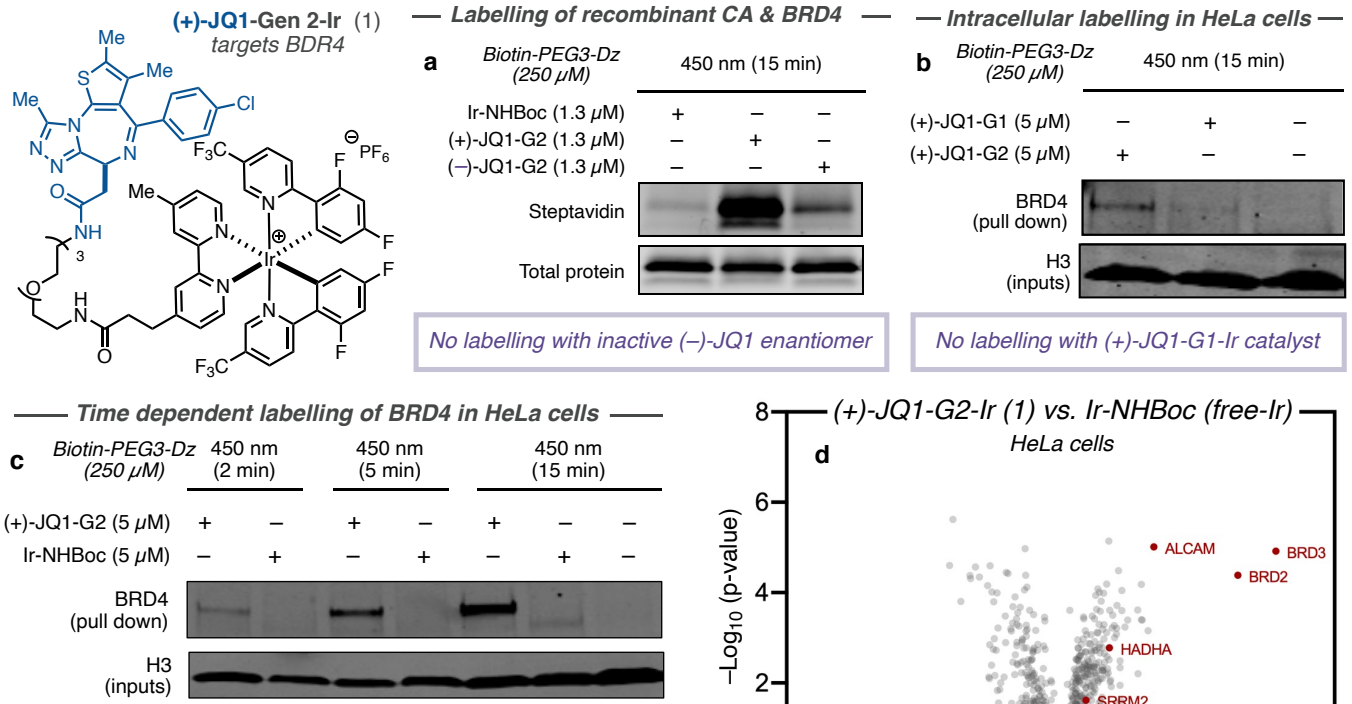

Labelling increases with time due to signal amplification Conclusive target ID using $\mu$ Map by chemoproteomics

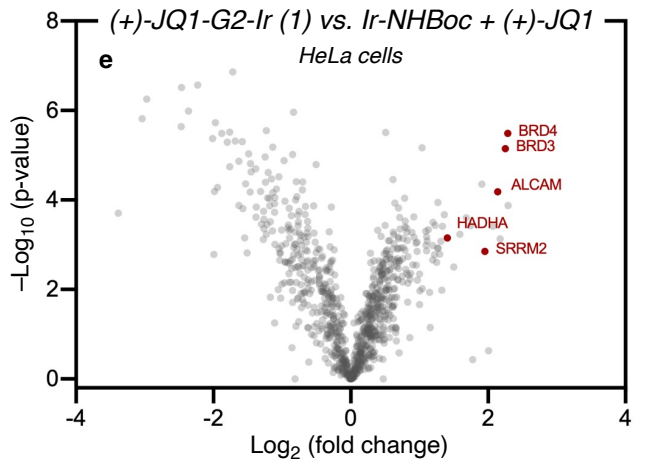

State-of-the-art UV photoaffinity labelling using JQ1-Dz-alkyne<smiles>C=CCCC1(CCNC(=O)CC2N=C(c3ccc(Cl)cc3)NC(C)=NN2)CN=N1</smiles>

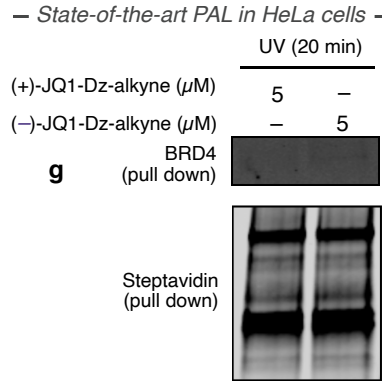

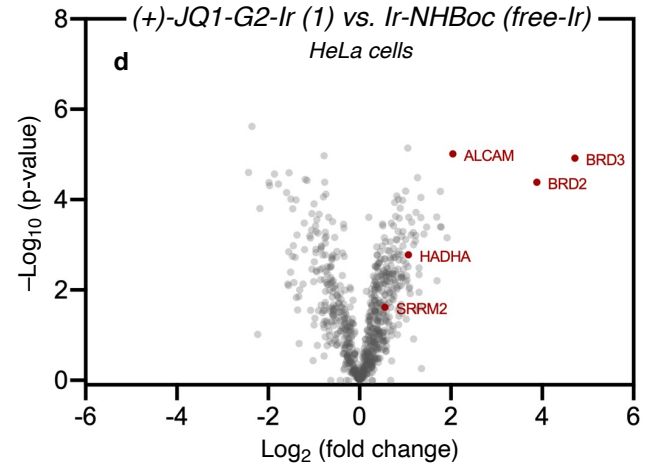
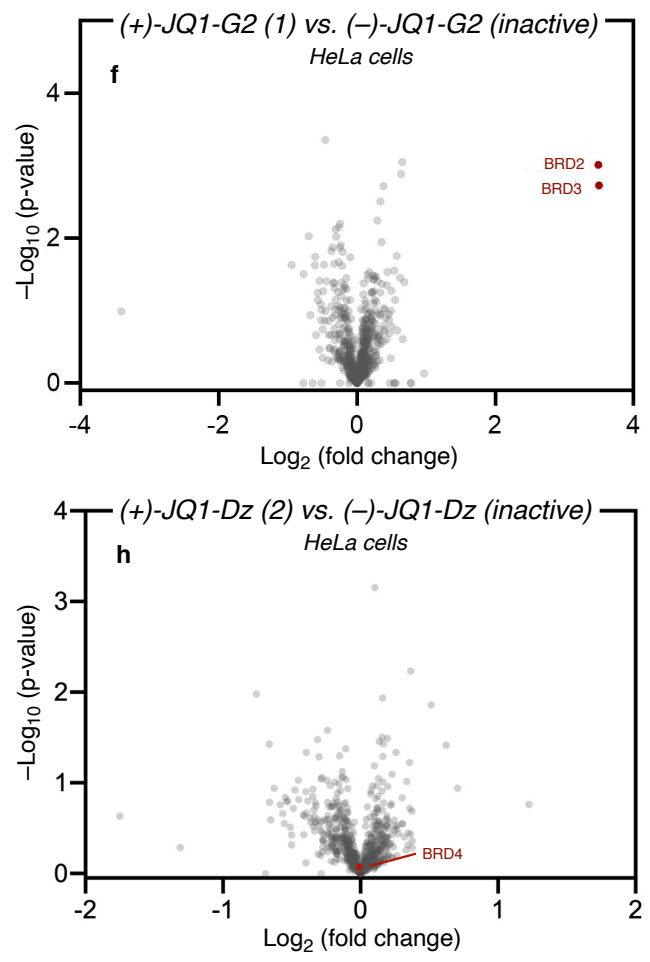

(+)-JQ1-Dz-alkyne (2) photoaffinity probe State-of-the-art
Scheme 2. Development of photocatalytic target ID platform for interactome mapping of $(+)-J Q 1$ in HeLa cells. Structure of JQ1-based photocatalyst conjugate and state-ofthe-art PAL probe. a, Labelling of recombinant BRD4 protein vs. spectator protein carbonic anhydrase using free iridium-, (+)-JQ1-, and (-)JQ1-probe by immunostaining with streptavidin. b, Comparing permeability of G1 and G2-based (+)-JQ1 probes following irradiation in $\mathrm{HeLa}$ cells, streptavidin bead enrichment, and immunostaining with antiBRD4. c, BRD4 labelling increases over time (2 min, $5 \mathrm{~min}$, and $15 \mathrm{~min}$ irradiation) through photocatalytic signal amplification using (+)-JQ1G2 probe in HeLa cells following streptavidin bead enrichment and immunostaining with anti-BRD4. d, TMT-based quantitative chemoproteomic analysis of JQ1-labelling in HeLa cells comparing intracellular labelling by $(+)-J Q 1-G 2$ catalysts and unconjugated iridium catalyst (control) reveals BRD proteins as highly enriched in addition to known JQ1 off-targets. e, TMT-based quantitative chemoproteomic analysis of JQ1-labelling in HeLa cells comparing intracellular labelling by (+)JQ1-G2 catalysts and unconjugated iridium catalyst $+(+)-J Q 1$ (control). f, TMT-based quantitative chemoproteomic analysis of JQ1-labelling in HeLa cells comparing intracellular labelling between active (+)-JQ1G2 and inactive (-)-JQ1-G2 catalysts reveals only $(+)$-isomer labels BRD proteins. g, State-of-the-art PAL employing active (+)-JQ1- and inactive (-)-JQ1-Dz-alkyne probes reveals no selective enrichment of BRD4 by western blotting despite broad biotinylation visible by immunostaining with streptavidin. $\mathbf{h}$, TMT-based quantitative chemoproteomic analysis in HeLa cells comparing state-of-the-art PAL employing active (+)-JQ1- and inactive (-)JQ1-Dz-alkyne probes reveals no enrichment of BRD proteins.

No conclusive target ID by quantitative chemoproteomics using state-of-the-art photoaffinity labelling

require low-yielding downstream 'click' processing ${ }^{19}$. Moreover, intracellular labelling technologies are often hampered by low cell-permeability leading to high background signal ${ }^{5}$. We therefore reasoned that the development of a catalytic target ID technology that separated the drug molecule from the reactive warhead could overcome these challenges through multiple labelling events leading to signal amplification (Scheme 1b).

We recently disclosed a novel antibody-based proximity labelling platform for cell surface microenvironment elucidation, termed $\mu \mathrm{Map}^{27}$. This method relies upon the activation of diazirine molecules in close proximity to a 
set of photocatalysts appended to an antibody via Dexter energy transfer. Inspired by this unique activation mode, we questioned whether such a tactic could be leveraged for small molecule target ID through the incorporation of a photocatalyst onto a bioactive small molecule. However, at the outset of the investigation, we were cognizant of several challenges inherent in developing such a technology, such as catalyst cell permeability and biocompatibility, ease of chemical manipulation, retention of biological activity, and labelling efficiency (given each antibody contained an average of 6-8 photocatalysts). However, we reasoned that by 'switching on' catalysis through visible light activation, labelling could be controlled both spatially and temporally, bypassing intrinsic reactivity problems and enabling the identification of novel targets across numerous drug discovery programs.

We began by investigating cell permeability: employing a halotag-based chaser assay off-competing a TAMRA dye in HEK293T cells, we identified that our previous catalyst design (Gen 1) was impermeable by virtue of its neutral net charge and two carboxylic acid residues (Scheme 1d). Through screening different photocatalyst structures, we realized that Ir-catalysts containing both the $\mathrm{dFCF}_{3}$-phenyl pyridine moiety and 4,4-dialkyl bpy ligand were crucial in achieving the necessary triplet energy ${ }^{27}$. Pleasingly, by removing the carboxylic acid groups, the cationic photocatalyst (Gen 2) was rendered cell permeable (Scheme 1d). With this in mind, we evaluated conjugation handles based around the 4,4-dMebpy ligand, opting for a distal carboxylic acid to enable facile amide coupling. Importantly, our G2-iridium catalyst can be accessed on gram-scale and be readily conjugated to a range of linkers and complex small molecules (vide infra).

Confident in our ability to access almost any Ir-drug conjugate, we initiated our target ID campaign with the validated epigenetic tool compound (+)-JQ1 ${ }^{28}$. A potent inhibitor of the BET family of bromodomain proteins (BRD2/3/4), several JQ1 structural analogues are in clinical trials for a variety of cancers including NUT midline carcinoma $^{29}$. We prepared the corresponding (+)-JQ1-G2 conjugate (1) (Scheme 2) and validated target engagement in vitro with recombinant BRD4 in a competition assay vs. bovine carbonic anhydrase (CA). An equimolar amount of CA and BRD4 was treated with (+)-JQ1-G2 probe (1) and an excess of diazirine-PEG3-biotin prior to irradiation at $450 \mathrm{~nm}$. Labelling intensity was measured by western blotting with a streptavidin stain. Pleasingly, these preliminary experiments revealed a 20 -fold increase in labelling for BRD4 vs. CA compared to the unconjugated (free) photocatalyst (Scheme 2a). Importantly, the (-)-JQ1-G2 conjugate, which is known to not bind BRD428, showed significantly reduced labelling, demonstrating that labelling is as a result of a ligand/protein binding event (Scheme 2c, A). In addition, we were able to confirm this through microscale thermophoresis (MST), where the addition of the Ir-catalyst made only a minor impact on the binding constant (Figure S1).

Based on these results, we sought to apply this method to live cells. We treated HeLa cells with $5 \mu \mathrm{M}(+)-J Q 1-$ Gen 2 (1) for $3 \mathrm{~h}$ before the addition of $250 \mu \mathrm{M}$ Dz-PEG3-Biotin and subsequent 15 min irradiation $(450 \mathrm{~nm})$. Following lysis and streptavidin-bead enrichment, western blot analysis with anti-BRD4 showed a clear labelling of the target protein compared to DMSO control (Figure S2). In line with previous findings, the corresponding $(+)$ JQ1-Gen 1 catalyst, while demonstrating similar in vitro labelling capability, showed no such enrichment of the target protein in cells (Scheme 2b). Consistent with our hypothesis, the intensity of labelling was found to be linearly related to irradiation time, demonstrating the photocatalytic signal amplification and temporal control offered by the $\mu$ Map platform (Scheme 2c). This was also observable by confocal microscopy, wherein the degree of biotinylation imparted by increased significantly over time (Figure S3). Encouraged by our western blot validation data, we moved to TMT-based quantitative chemoproteomics in order to more completely assess the interactome of $(+)$ JQ1. To our delight, by comparing the labelling by (+)-JQ1-Gen 2 (1) vs. unconjugated (free) photocatalyst in HeLa cells, we observed several BRD proteins as the most enriched, although the precise identity of which however remains difficult to ascertain due to structural homology (Scheme 2d). We also identified two previously annotated (+)-JQ1 off-targets, HADHA ${ }^{30}$ and SRRM2 ${ }^{31}$. ALCAM (CD166), a transmembrane glycoprotein, was also identified as being significantly enriched, but currently has no reported interaction with (+)-JQ1. CD166 exerts a pro- 
bioRxiv preprint doi: https://doi.org/10.1101/2021.08.02.454797; this version posted August 2, 2021. The copyright holder for this preprint (which was not certified by peer review) is the author/funder, who has granted bioRxiv a license to display the preprint in perpetuity. It is made available under aCC-BY-NC-ND 4.0 International license.

carcinogenic role via the inhibition of transcription factors along the FOXO/AKT axis and is considered a novel therapeutic target for liver cancer ${ }^{32}$. Interestingly, BET inhibition by (+)-JQ1 has been shown to upregulate expression of FOXO1, although the mechanism remains unclear ${ }^{33}$. In order to evaluate whether protein enrichment was as a direct result of labelling or up-regulation by virtue of the presence of (+)-JQ1, we repeated the experiment with
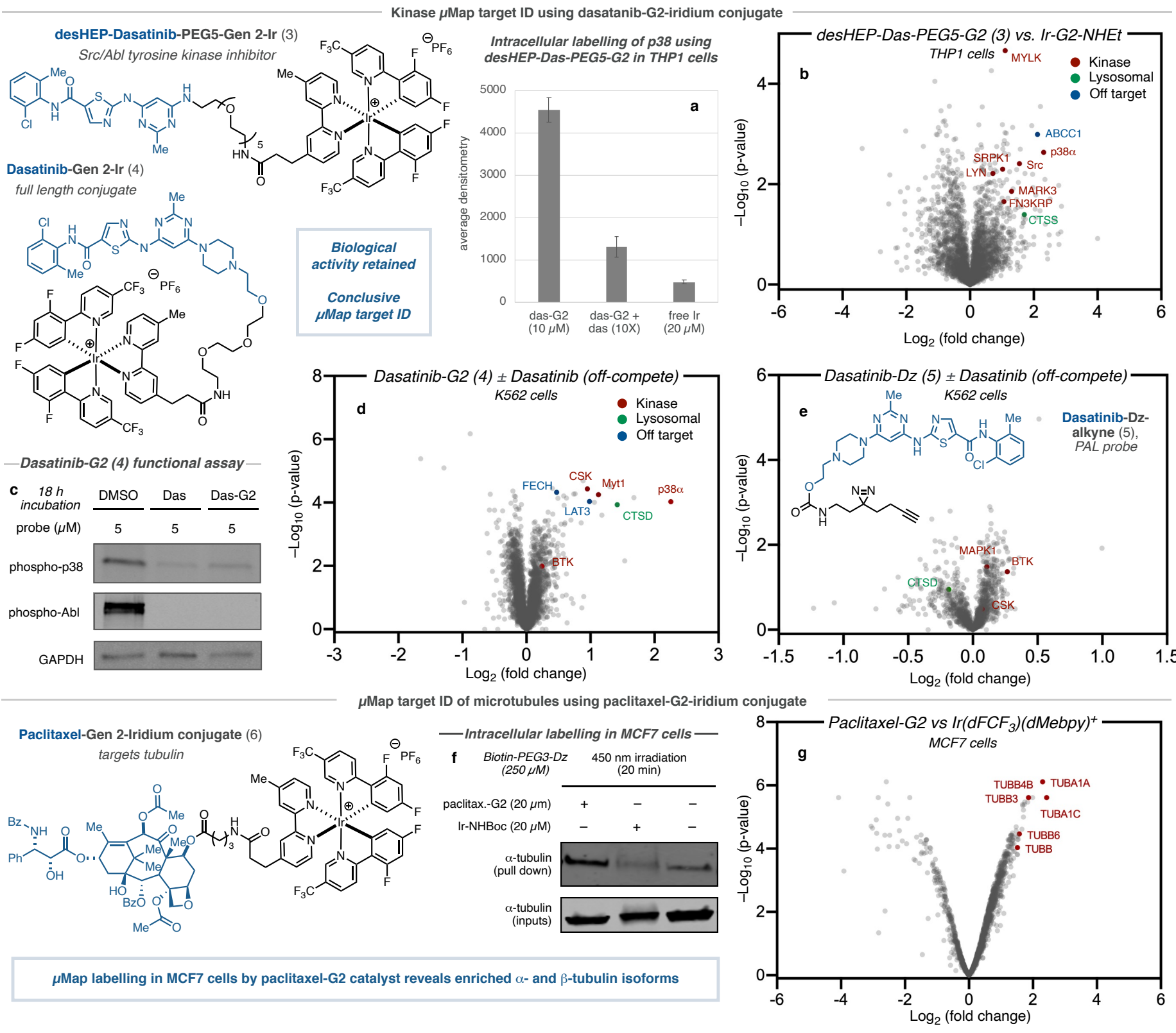

Scheme 3. Intracellular photocatalytic target ID and interactome mapping of dasatinib and paclitaxel. a, Enrichment of p38 by western blot for labelling using desHEP-dasatinib-PEG5-G2 labelling in THP1 cells. b, Label free proteomic analysis in THP1 cells comparing intracellular labelling by desHEP-dasatinib-PEG5-G2 catalyst vs. Ir-G2-NHEt reveals enrichment of several kinases (red), as well as lysosomal proteins (green) and off-targets (blue). c, Kinase activity assays reveals dasatinib-G2 retains inhibition activity against Abl and p38, as well as general tyrosine phosphorylation, in K562 cells. d, TMT-based quantitative chemoproteomic analysis in K562 cells comparing intracellular labelling by dasatinib-G2 catalyst vs. dasatinib-G2 + dasatinib (off-compete control) reveals enrichment of several kinases (red), as well as lysosomal proteins (green) and established off-targets (blue). e, TMT-based quantitative chemoproteomic analysis in K562 cells comparing intracellular labelling by dasatinib-Dz-alkyne (PAL probe) vs. off-compete control does not reveal enrichment of kinases suitable for conclusive target ID. f, Initial western blot studies for paclitaxel-G2 labelling in MCF7 cells following irradiation and streptavidin bead enrichment reveals significant enrichment of a-tubulin by immunostaining compared to unconjugated iridium and DMSO controls. g, TMT-based quantitative chemoproteomic analysis in MCF7 cells comparing intracellular labelling by paclitaxel-G2 catalyst and unconjugated iridium catalyst (control) reveals enrichment of several tubulin isoforms. 
an equivalent of (+)-JQ1 in the free iridium control (Scheme 2e). Upon chemoproteomic analysis we found that CD166 was similarly enriched, indicating that it may be a putative off-target binder of (+)-JQ1, although further biological validation is required. We further compared the interactomes of the enantiomers of JQ1-G2 and found the active (+) enantiomer, (1), delivered BRD2/3/4 as top hits, and while CD166 was detected it was not enriched, indicating that binding may not be affected by the stereogenic center (Scheme 2e). In contrast to these data, the same analysis using classical UV-based PAL employing (+)-JQ-Dz-alkyne (2) ${ }^{34}$, in our hands, did not lead to enrichment of BRD proteins by western blot (Scheme $2 \mathrm{~g}$ ) or chemoproteomic analysis (Scheme $2 \mathrm{~h}$ ).

The dual Src/Abl tryrosine kinase inhibitor dasatinib displays significant antileukemic effects against various imatinib-resistant mutants ${ }^{35}$. However, despite well-documented BCR/ABL inhibition, its precise downstream cellular MOA remains to be fully understood. While the dasatinib interactome has been previously characterized ${ }^{16}$, most methods have been performed with recombinant protein or in cell-lysate; live cell data is typically restricted to kinase-based assays that measure downstream phosphorylation or residence at engineered kinase constructs, which can be challenging to deconvolute and fail to identify non-kinase based off targets ${ }^{36-39}$.

As previous studies have demonstrated difficulties in maintaining potency and cell-permeability using dasatinibderived probes ${ }^{16}$, we started by synthesizing three truncated (des $\underline{H} y d r o x y \underline{E}$ thylPiperazinyl)-dasatinib iridium conjugates using our cell-permeable Ir-G2 catalyst with varying PEG linker lengths $(\mathrm{n}=3-5)$ (3) (Scheme 3, top). Gratifyingly, upon subjection of the desHEP-dasatinib-G2 conjugates (3) $(5 \mu \mathrm{M})$ to our standard $\mu$ Map protocol, all of the conjugates revealed enrichment of p38 (MAP kinase) by western blot analysis compared to off-compete (4X dasatinib) controls in THP1 cells (Figure S4). As the corresponding PEG5-G2 conjugate showed the greatest enrichment (3.5X enrichment vs. off-compete and 9.5X enrichment vs. free-Ir) (Scheme 3a), we undertook label free proteomic analysis of these reactions revealing significant enrichment of p38 $\alpha$ (Figure S5), which has been shown to play a critical role in its antileukemic properties ${ }^{40}$, as well as several other established kinase interactors including Src and Lyn (Scheme 3b) ${ }^{41}$. Furthermore, we identified multidrug resistance transporter ABCC1 amongst the most enriched proteins - an important off target; understanding the interaction between drug molecules and efflux transporters is an important consideration in many drug discovery efforts ${ }^{42}$. Lysosomal sequestration of dasatinib $^{43}$, due to its lipophilic and weakly basic properties, was evident by the presence of cathepsin S (CTSS) amongst the most enriched proteins. Encouraged by these initial results, we turned our attention to the underexplored full dasatinib-PEG3-G2 catalyst (4), which retains the 2-hydroxyethylpiperazine tail. Importantly, we found a similar kinase inhibition profile against $\mathrm{p} 38$, in addition to Abl, by evaluation of downstream phosphorylation in $\mathrm{Ph}+\mathrm{K} 562$ cells, compared to the parent drug, again highlighting the compatibility of the iridium photocatalyst towards maintaining biological function and cell permeability (Scheme 3c) (Figure S6). Gratifyingly, subjection of our $\mu$ Map labelling to TMT-based chemoproteomics revealed extensive enrichment of p38 $\alpha$ as well as Myt1 and CSK kinases, both well-established binders of dasatinib (Scheme 3d) ${ }^{39}$. Moreover, known kinase off target ferrochelatase $(\mathrm{FECH})^{44}$ was also significantly enriched, alongside large amino acid transporter (LAT3) ${ }^{39}$. Similarly, lysosomal protein cathepsin D (CTSD) was amongst the most enriched proteins. Notably, in our hands, state-ofthe-art photoaffinity labelling, employing dasatinib-diazirine-alkyne (5), revealed only trace enrichment of CSK and the kinases BTK and MAPK1 (BTK was found to be similarly enriched by $\mu$ Map) (Scheme 3e).

The anti-cancer properties of the natural product paclitaxel (Taxol) have been proposed to be derived from binding to microtubules, leading to stabilization and mitotic arrest; however, the full extent of its mechanism remains unclear $^{45}$. Based on its widespread use and intriguing mechanism, we prepared the corresponding paclitaxel-Gen 2iridium conjugate (6) (Scheme 3, bottom) and assessed its cellular activity. Through a series of cell proliferation assays we found that our paclitaxel-G2 conjugate displayed similar anti-proliferative properties as the native compound, suggesting that the pendent Ir-catalyst did not disrupt the native function of paclitaxel (Figure S7). Encouraged by this, we proceeded to study the efficiency of labelling in the breast cancer cell line MCF7. Following our 
standard $\mu$ Map protocol with $20 \mu \mathrm{M}$ paclitaxel-G2 conjugate (6) for $3 \mathrm{~h}$, western blot analysis with anti- $\alpha$-tubulin showed clear labelling of the target protein compared both the free iridium and DMSO controls (Scheme 3f). Subjection of our $\mu$ Map labelling to TMT-based chemoproteomics revealed extensive labelling of tubulin isotypes $\alpha$ Ia, $\beta I I I, \beta I V b$, and $\alpha \mathrm{Ic}$ (Scheme $3 \mathrm{~g}$ ), which is in good agreement with previous photoaffinity labelling studies on extracted tubulin ${ }^{46}$.

Having established the efficacy of $\mu$ Map target ID for intracellular proteins, we turned our attention to the cell surface. The exceedingly low abundance, lack of exposed residues, and aggregation-prone hydrophobic domains oftentimes confounds the detection and manipulation of membrane proteins, rendering target ID unfeasible ${ }^{19,47,48}$. These challenges are exacerbated when combined with the high background labelling, poor sensitivity, and low cross-linking yields systemic in PAL campaigns. We therefore felt that our $\mu$ Map target ID platform was ideally placed to tackle these challenges by virtue of our catalytic signal amplification. We chose the adenosine receptor $\mathrm{A}_{2 \mathrm{a}}$ (ADORA2A) as an exemplar membrane target. This GPCR has become an important target for immunotherapy $^{49}$, but critically, has never been identified through live cell chemoproteomics ${ }^{50,51}$. Using a reported ligand for ADORA2A that binds from the extracellular face, $\mathrm{SCH} 58261^{52}$, we prepared both a tethered diazirine-conjugate, SCH58261-Dz (7), and an Ir-conjugate based on the more hydrophilic G1 catalyst, SCH58261-G1 (8); the low cell

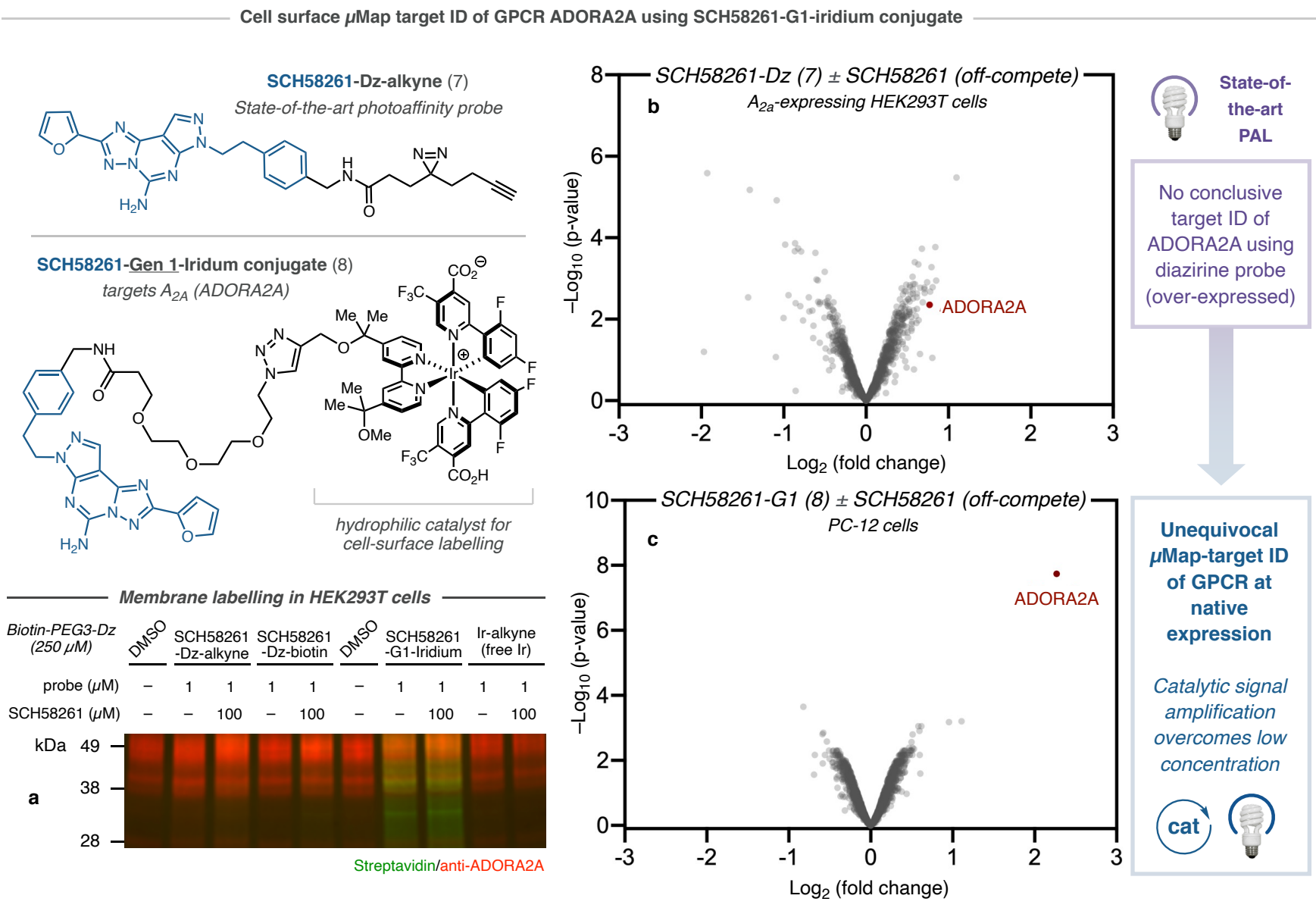

Scheme 4. Extracellular photocatalytic target ID of GPCR ADORA2A using SCH58261 at native expression. a, Structure of SCH58261-based Dzalkyne probe for PAL labelling and iridium conjugate based on hydrophilic G1 photocatalyst. b, Initial western blot studies for SCH58261-G1 labelling in $\mathrm{A}_{2 \mathrm{a}}$-expressing HEK293T cells following irradiation and streptavidin bead enrichment reveals significant biotinylation by immunostaining compared to unconjugated iridium and DMSO controls, in addition to PAL labelling. c, TMT-based quantitative chemoproteomic analysis in $\mathrm{A}_{2 \mathrm{a}}$-expressing HEK293T cells comparing extracellular labelling by SCH58261-Dz-alkyne vs. SCH58261-Dz-alkyne + SCH58261 (off-compete control) reveals inconclusive target ID of ADORA2A. d, TMT-based quantitative chemoproteomic analysis in PC-12 cells comparing extracellular labelling by SCH58261-G1 catalyst vs. SCH58261G1 + SCH58261 (off-compete control) reveals significant enrichment of ADORA2A. 
permeability affording a higher effective concentration of photocatalyst probe on the cell surface (Scheme 4a). Photocatalytic labelling applied to $\mathrm{A}_{2 \mathrm{a}}$-expressing HEK293T cells, followed by western blot visualization, revealed a stark difference in labelling between the SCH58261-G1 (8) and the corresponding off-compete controls (Scheme 4b). Tandem mass tag (TMT)-based chemoproteomic analysis of these reactions confirmed our initial result, with our photocatalytic-labelling method using SCH58261-G1 (8) showing a 10-fold enrichment for ADORA2A with respect to off-competing with the parent SCH58261 ligand, and $>20$-fold enrichment versus free-Ir photocatalyst (Figure S8a). In contrast, PAL using SCH58261-Dz (7), showed poor enrichment of ADORA2A by quantitative chemoproteomics (Scheme 4c), in line with western blot data (Figure S8b). Based on the degree of enrichment in $\mathrm{A}_{2 \mathrm{a}}$-expressing HEK293T cells, we were keen to ascertain how the $\mu$ Map target ID platform performed at native levels of membrane protein concentration, wherein classical PAL remains extremely challenging. Remarkably, photocatalytic labelling using SCH58261-G1 (8) in PC-12 cells, which have previously been validated to natively express $\mathrm{A}_{2 \mathrm{a}}{ }^{53}$, revealed similarly high levels of enrichment for the target protein ADORA2A - highlighting the signal amplification conferred by the $\mu$ Map platform (Scheme $4 \mathrm{~d}$ ). Finally, we further validated this labelling technology by identifying the long chain fatty acid receptor GPR40, an important anti-diabetic therapeutic target, using the small molecule probe MK-8666, further expanding the repertoire of $\mu$ Map membrane target ID (Figure S9-S12) ${ }^{54}$.

In conclusion, we describe a general platform for photocatalytic target ID that utilizes cell-penetrating iridium conjugated-small molecules, which can bind protein targets, to locally activate proximal diazirines via Dexter energy transfer. The catalytic signal amplification conferred by $\mu$ Map target ID has allowed for the identification of multiple protein targets and off targets across multiple drug classes and cellular compartments where established PAL have not been successful. As such, we envision that $\mu$ Map target ID will find immediate use in providing a deeper biological understanding of efficacy target networks, quickly revealing off-target pharmacology, and ultimately driving pharmacotherapy forward against novel targets within drug discovery programmes in both academic and industrial settings.

\section{Acknowledgments.}

Research reported in this publication was supported by the NIH National Institute of General Medical Sciences (R01- GM103558-03) and gifts from Merck \& Co., Inc., Kenilworth, New Jersey, USA. ADT would like to thank the European Union's Horizon 2020 research and innovation programme under Marie Sklodowska-Curie Grant Agreement No.891458. JBG acknowledges the NIH for a postdoctoral fellowship (F32-GM133133-01). JVO acknowledges the National Science Foundation Graduate Research Fellowship Programme under Grant No. (DGE1656466). Any opinions, findings, and conclusions or recommendations expressed in this material are those of the authors and do not necessarily reflect the views of the National Science Foundation. The authors thank Saw Kyin and Henry H. Shwe at the Princeton Proteomics Facility. The authors thank Brande Thomas-Fowlkes and Xiaoping Zhang (MRL, Merck \& Co., Inc, Kenilworth, NJ, USA), for providing GPR40-HEK cells and running IP-1 assay, respectively. HEK-hA2aR cell line was received as a gift from Jeremy Presland (MRL, Merck \& Co., Inc, Boston, MA, USA). We acknowledge the use of Princeton's Imaging and Analysis Center, which is partially supported by the Princeton Center for Complex Materials, a National Science Foundation/Materials Research Science and Engineering Centers programme (DMR-1420541). We also acknowledge V. G. Vendavasi and the use of Princeton's Biophysics Core Facility. We thank Antony Burton for assistance in performing confocal microscopy. We thank T. W. Muir and members of the Muir Laboratory for their advice and analytical support.

\section{Author Affiliations and Contributions}

${ }^{1}$ Merck Center for Catalysis at Princeton University, Princeton, NJ 08544, USA.

${ }^{2}$ Discovery Chemistry, Merck \& Co., Inc, Kenilworth NJ 07033, USA 
${ }^{3}$ Department of Chemistry, Princeton University, Princeton, NJ 08544, USA

${ }^{4}$ Discovery Chemistry, Merck \& Co., Inc., Boston, MA 02115, USA.

${ }^{5}$ Merck Exploratory Science Center, Merck \& Co., Inc., Cambridge, MA 02141, USA.

${ }^{6}$ Pharmacology, Merck \& Co., Inc., Kenilworth, NJ 07033, USA.

Author contributions: ADT, CPS, FPR-R, DLP, DWCM conceived the work. FPR-R, DLP, CPS, ADT, BXL, $\mathrm{BD}, \mathrm{JBG}, \mathrm{JVO}, \mathrm{AGS}, \mathrm{OOF}, \mathrm{RCO}$ designed and executed the experiments. PT, TR-R, KAR provided insight into experimental design. ADT, CPS, FPR-R, DLP, DWCM prepared this manuscript. *These authors contributed equally

Competing interest: A provisional U.S. patent has been filed by DWCM, ADT, CPS based in part on this work, 62/982,366; 63/076,658. International Application No. PCT/US2021/019959. DWCM declares an ownership interest, and ADT and CPS declare an affiliation interest, in the company Dexterity Pharma LLC, which has commercialized materials used in this work. DWCM declares an ownership interest in Penn PhD, which has commercialized materials used in this work.

Correspondence: ${ }^{\dagger}$ Corresponding authors. Correspondence and requests for materials should be addressed to D.W.C.M (dmacmill@princeton.edu), FPR-R (frances.rodriguez-rivera@merck.com), and DLP (dann_parker@merck.com).

\section{References}

(1) Chan, J. N. Y.; Nislow, C.; Emili, A. Recent Advances and Method Development for Drug Target Identification. Trends Pharmacol. Sci. 2010, 31 (2), 82-88. https://doi.org/https://doi.org/10.1016/j.tips.2009.11.002.

(2) Schenone, M.; Dančík, V.; Wagner, B. K.; Clemons, P. A. Target Identification and Mechanism of Action in Chemical Biology and Drug Discovery. Nat. Chem. Biol. 2013, 9 (4), 232-240. https://doi.org/10.1038/nchembio.1199.

(3) Hart, C. P. Finding the Target after Screening the Phenotype. Drug Discov. Today 2005, 10 (7), 513-519. https://doi.org/https://doi.org/10.1016/S1359-6446(05)03415-X.

(4) Williams, M. Target Validation. Curr. Opin. Pharmacol. 2003, 3 (5), 571-577. https://doi.org/https://doi.org/10.1016/j.coph.2003.06.001.

(5) Ziegler, S.; Pries, V.; Hedberg, C.; Waldmann, H. Target Identification for Small Bioactive Molecules: Finding the Needle in the Haystack. Angew. Chemie Int. Ed. 2013, 52 (10), 2744-2792. https://doi.org/10.1002/anie.201208749.

(6) Morgan, P.; Brown, D. G.; Lennard, S.; Anderton, M. J.; Barrett, J. C.; Eriksson, U.; Fidock, M.; Hamrén, B.; Johnson, A.; March, R. E.; Matcham, J.; Mettetal, J.; Nicholls, D. J.; Platz, S.; Rees, S.; Snowden, M. A.; Pangalos, M. N. Impact of a Five-Dimensional Framework on R\&D Productivity at AstraZeneca. Nat. Rev. Drug Discov. 2018, 17 (3), 167-181. https://doi.org/10.1038/nrd.2017.244.

(7) Schirle, M.; Bantscheff, M.; Kuster, B. Mass Spectrometry-Based Proteomics in Preclinical Drug Discovery. Chem. Biol. 2012, 19 (1), 72-84.

https://doi.org/https://doi.org/10.1016/j.chembiol.2012.01.002.

(8) Wagner, B. K.; Clemons, P. A. Connecting Synthetic Chemistry Decisions to Cell and Genome Biology Using Small-Molecule Phenotypic Profiling. Curr. Opin. Chem. Biol. 2009, 13 (5), 539-548. 
https://doi.org/https://doi.org/10.1016/j.cbpa.2009.09.018.

(9) Butcher, E. C.; Berg, E. L.; Kunkel, E. J. Systems Biology in Drug Discovery. Nat. Biotechnol. 2004, 22 (10), 1253-1259. https://doi.org/10.1038/nbt1017.

(10) Schürmann, M.; Janning, P.; Ziegler, S.; Waldmann, H. Small-Molecule Target Engagement in Cells. Cell Chem. Biol. 2016, 23 (4), 435-441. https://doi.org/https://doi.org/10.1016/j.chembiol.2016.03.008.

(11) Simon, G. M.; Niphakis, M. J.; Cravatt, B. F. Determining Target Engagement in Living Systems. Nat. Chem. Biol. 2013, 9 (4), 200-205. https://doi.org/10.1038/nchembio.1211.

(12) Kawatani, M.; Osada, H. Affinity-Based Target Identification for Bioactive Small Molecules. Medchemcomm 2014, 5 (3), 277-287. https://doi.org/10.1039/C3MD00276D.

(13) Hill, J. R.; Robertson, A. A. B. Fishing for Drug Targets: A Focus on Diazirine Photoaffinity Probe Synthesis. J. Med. Chem. 2018, 61 (16), 6945-6963. https://doi.org/10.1021/acs.jmedchem.7b01561.

(14) Ge, S.-S.; Chen, B.; Wu, Y.-Y.; Long, Q.-S.; Zhao, Y.-L.; Wang, P.-Y.; Yang, S. Current Advances of Carbene-Mediated Photoaffinity Labeling in Medicinal Chemistry. RSC Adv. 2018, 8 (51), 29428-29454. https://doi.org/10.1039/C8RA03538E.

(15) Smith, E.; Collins, I. Photoaffinity Labeling in Target- and Binding-Site Identification. Future Med. Chem. 2015, 7 (2), 159-183. https://doi.org/10.4155/fmc.14.152.

(16) Shi, H.; Zhang, C.-J.; Chen, G. Y. J.; Yao, S. Q. Cell-Based Proteome Profiling of Potential Dasatinib Targets by Use of Affinity-Based Probes. J. Am. Chem. Soc. 2012, 134 (6), 3001-3014. https://doi.org/10.1021/ja208518u.

(17) Zuhl, A. M.; Nolan, C. E.; Brodney, M. A.; Niessen, S.; Atchison, K.; Houle, C.; Karanian, D. A.; Ambroise, C.; Brulet, J. W.; Beck, E. M.; Doran, S. D.; O’Neill, B. T.; am Ende, C. W.; Chang, C.; Geoghegan, K. F.; West, G. M.; Judkins, J. C.; Hou, X.; Riddell, D. R.; Johnson, D. S. Chemoproteomic Profiling Reveals That Cathepsin D Off-Target Activity Drives Ocular Toxicity of $\beta$-Secretase Inhibitors. Nat. Commun. 2016, 7 (1), 13042. https://doi.org/10.1038/ncomms13042.

(18) Ito, T.; Ando, H.; Suzuki, T.; Ogura, T.; Hotta, K.; Imamura, Y.; Yamaguchi, Y.; Handa, H. Identification of a Primary Target of Thalidomide Teratogenicity. Science 2010, 327 (5971), 1345 LP - 1350. https://doi.org/10.1126/science.1177319.

(19) Wright, M. H.; Sieber, S. A. Chemical Proteomics Approaches for Identifying the Cellular Targets of Natural Products. Nat. Prod. Rep. 2016, 33 (5), 681-708. https://doi.org/10.1039/C6NP00001K.

(20) Park, J.; Koh, M.; Koo, J. Y.; Lee, S.; Park, S. B. Investigation of Specific Binding Proteins to Photoaffinity Linkers for Efficient Deconvolution of Target Protein. ACS Chem. Biol. 2016, 11 (1), 44-52. https://doi.org/10.1021/acschembio.5b00671.

(21) Hayashi, T.; Hamachi, I. Traceless Affinity Labeling of Endogenous Proteins for Functional Analysis in Living Cells. Acc. Chem. Res. 2012, 45 (9), 1460-1469. https://doi.org/10.1021/ar200334r.

(22) Yamaura, K.; Kiyonaka, S.; Numata, T.; Inoue, R.; Hamachi, I. Discovery of Allosteric Modulators for GABAA Receptors by Ligand-Directed Chemistry. Nat. Chem. Biol. 2016, 12 (10), 822-830. https://doi.org/10.1038/nchembio.2150.

(23) Fujishima, S.; Yasui, R.; Miki, T.; Ojida, A.; Hamachi, I. Ligand-Directed Acyl Imidazole Chemistry for Labeling of Membrane-Bound Proteins on Live Cells. J. Am. Chem. Soc. 2012, 134 (9), 3961-3964. https://doi.org/10.1021/ja2108855.

(24) Herner, A.; Marjanovic, J.; Lewandowski, T. M.; Marin, V.; Patterson, M.; Miesbauer, L.; Ready, D.; Williams, J.; Vasudevan, A.; Lin, Q. 2-Aryl-5-Carboxytetrazole as a New Photoaffinity Label for Drug Target Identification. J. Am. Chem. Soc. 2016, 138 (44), 14609-14615. https://doi.org/10.1021/jacs.6b06645. 
(25) Sato, S.; Nakamura, H. Ligand-Directed Selective Protein Modification Based on Local Single-ElectronTransfer Catalysis. Angew. Chemie Int. Ed. 2013, 52 (33), 8681-8684.

https://doi.org/10.1002/anie.201303831.

(26) Sato, S.; Tsushima, M.; Nakamura, H. Target-Protein-Selective Inactivation and Labelling Using an Oxidative Catalyst. Org. Biomol. Chem. 2018, 16 (34), 6168-6179. https://doi.org/10.1039/C8OB01484A.

(27) Geri, J. B.; Oakley, J. V.; Reyes-Robles, T.; Wang, T.; McCarver, S. J.; White, C. H.; Rodriguez-Rivera, F. P.; Parker, D. L.; Hett, E. C.; Fadeyi, O. O.; Oslund, R. C.; MacMillan, D. W. C. Microenvironment Mapping via Dexter Energy Transfer on Immune Cells. Science 2020, 367 (6482), 1091-1097. https://doi.org/10.1126/science.aaz5074.

(28) Filippakopoulos, P.; Qi, J.; Picaud, S.; Shen, Y.; Smith, W. B.; Fedorov, O.; Morse, E. M.; Keates, T.; Hickman, T. T.; Felletar, I.; Philpott, M.; Munro, S.; McKeown, M. R.; Wang, Y.; Christie, A. L.; West, N.; Cameron, M. J.; Schwartz, B.; Heightman, T. D.; La Thangue, N.; French, C. A.; Wiest, O.; Kung, A. L.; Knapp, S.; Bradner, J. E. Selective Inhibition of BET Bromodomains. Nature 2010, 468 (7327), 10671073. https://doi.org/10.1038/nature09504.

(29) Shorstova, T.; Foulkes, W. D.; Witcher, M. Achieving Clinical Success with BET Inhibitors as AntiCancer Agents. Br. J. Cancer 2021, 124 (9), 1478-1490. https://doi.org/10.1038/s41416-021-01321-0.

(30) Kurzawa, N.; Becher, I.; Sridharan, S.; Franken, H.; Mateus, A.; Anders, S.; Bantscheff, M.; Huber, W.; Savitski, M. M. A Computational Method for Detection of Ligand-Binding Proteins from Dose Range Thermal Proteome Profiles. Nat. Commun. 2020, 11 (1), 5783. https://doi.org/10.1038/s41467-020-195298 .

(31) Tyler, D. S.; Vappiani, J.; Cañeque, T.; Lam, E. Y. N.; Ward, A.; Gilan, O.; Chan, Y.-C.; Hienzsch, A.; Rutkowska, A.; Werner, T.; Wagner, A. J.; Lugo, D.; Gregory, R.; Ramirez Molina, C.; Garton, N.; Wellaway, C. R.; Jackson, S.; MacPherson, L.; Figueiredo, M.; Stolzenburg, S.; Bell, C. C.; House, C.; Dawson, S.-J.; Hawkins, E. D.; Drewes, G.; Prinjha, R. K.; Rodriguez, R.; Grandi, P.; Dawson, M. A. Click Chemistry Enables Preclinical Evaluation of Targeted Epigenetic Therapies. Science 2017, eaal2066. https://doi.org/10.1126/science.aal2066.

(32) Yu, W.; Wang, J.; Ma, L.; Tang, X.; Qiao, Y.; Pan, Q.; Yu, Y.; Sun, F. CD166 Plays a Pro-Carcinogenic Role in Liver Cancer Cells via Inhibition of FOXO Proteins through AKT. Oncol Rep 2014, 32 (2), 677683. https://doi.org/10.3892/or.2014.3226.

(33) Tan, Y.; Wang, L.; Du, Y.; Liu, X.; Chen, Z.; Weng, X.; Guo, J.; Chen, H.; Wang, M.; Wang, X. Inhibition of BRD4 Suppresses Tumor Growth in Prostate Cancer via the Enhancement of FOXO1 Expression. Int J Oncol 2018, 53 (6), 2503-2517. https://doi.org/10.3892/ijo.2018.4577.

(34) Li, Z.; Wang, D.; Li, L.; Pan, S.; Na, Z.; Tan, C. Y. J.; Yao, S. Q. "Minimalist” Cyclopropene-Containing Photo-Cross-Linkers Suitable for Live-Cell Imaging and Affinity-Based Protein Labeling. J. Am. Chem. Soc. 2014, 136 (28), 9990-9998. https://doi.org/10.1021/ja502780z.

(35) Shah, N. P.; Tran, C.; Lee, F. Y.; Chen, P.; Norris, D.; Sawyers, C. L. Overriding Imatinib Resistance with a Novel ABL Kinase Inhibitor. Science 2004, 305 (5682), 399 LP - 401.

https://doi.org/10.1126/science.1099480.

(36) Karaman, M. W.; Herrgard, S.; Treiber, D. K.; Gallant, P.; Atteridge, C. E.; Campbell, B. T.; Chan, K. W.; Ciceri, P.; Davis, M. I.; Edeen, P. T.; Faraoni, R.; Floyd, M.; Hunt, J. P.; Lockhart, D. J.; Milanov, Z. V; Morrison, M. J.; Pallares, G.; Patel, H. K.; Pritchard, S.; Wodicka, L. M.; Zarrinkar, P. P. A Quantitative Analysis of Kinase Inhibitor Selectivity. Nat. Biotechnol. 2008, 26 (1), 127-132. https://doi.org/10.1038/nbt1358.

(37) Hantschel, O.; Rix, U.; Schmidt, U.; Bürckstümmer, T.; Kneidinger, M.; Schütze, G.; Colinge, J.; Bennett, K. L.; Ellmeier, W.; Valent, P.; Superti-Furga, G. The Btk Tyrosine Kinase Is a Major Target of the BcrAbl Inhibitor Dasatinib. Proc. Natl. Acad. Sci. 2007, 104 (33), 13283-13288. 
(38) Vasta, J. D.; Corona, C. R.; Wilkinson, J.; Zimprich, C. A.; Hartnett, J. R.; Ingold, M. R.; Zimmerman, K.; Machleidt, T.; Kirkland, T. A.; Huwiler, K. G.; Ohana, R. F.; Slater, M.; Otto, P.; Cong, M.; Wells, C. I.; Berger, B.-T.; Hanke, T.; Glas, C.; Ding, K.; Drewry, D. H.; Huber, K. V. M.; Willson, T. M.; Knapp, S.; Müller, S.; Meisenheimer, P. L.; Fan, F.; Wood, K. V; Robers, M. B. Quantitative, Wide-Spectrum Kinase Profiling in Live Cells for Assessing the Effect of Cellular ATP on Target Engagement. Cell Chem. Biol. 2018, 25 (2), 206-214.e11. https://doi.org/10.1016/j.chembiol.2017.10.010.

(39) Friedman Ohana, R.; Levin, S.; Hurst, R.; Rosenblatt, M. M.; Zimmerman, K.; Machleidt, T.; Wood, K. V; Kirkland, T. A. Streamlined Target Deconvolution Approach Utilizing a Single Photoreactive Chloroalkane Capture Tag. ACS Chem. Biol. 2021, 16 (2), 404-413. https://doi.org/10.1021/acschembio.0c00987.

(40) Dumka, D.; Puri, P.; Carayol, N.; Lumby, C.; Balachandran, H.; Schuster, K.; Verma, A. K.; Terada, L. S.; Platanias, L. C.; Parmar, S. Activation of the P38 Map Kinase Pathway Is Essential for the Antileukemic Effects of Dasatinib. Leuk. Lymphoma 2009, 50 (12), 2017-2029.

https://doi.org/10.3109/10428190903147637.

(41) Williams, N. K.; Lucet, I. S.; Klinken, S. P.; Ingley, E.; Rossjohn, J. Crystal Structures of the Lyn Protein Tyrosine Kinase Domain in Its Apo- and Inhibitor-Bound State. J. Biol. Chem. 2009, 284 (1), $284-291$. https://doi.org/https://doi.org/10.1074/jbc.M807850200.

(42) Gottesman, M. M.; Fojo, T.; Bates, S. E. Multidrug Resistance in Cancer: Role of ATP-Dependent Transporters. Nat. Rev. Cancer 2002, 2 (1), 48-58. https://doi.org/10.1038/nrc706.

(43) Ruzickova, E.; Skoupa, N.; Dolezel, P.; Smith, D. A.; Mlejnek, P. The Lysosomal Sequestration of Tyrosine Kinase Inhibitors and Drug Resistance. Biomolecules 2019, 9 (11), 675 https://doi.org/10.3390/biom9110675.

(44) Klaeger, S.; Gohlke, B.; Perrin, J.; Gupta, V.; Heinzlmeir, S.; Helm, D.; Qiao, H.; Bergamini, G.; Handa, H.; Savitski, M. M.; Bantscheff, M.; Médard, G.; Preissner, R.; Kuster, B. Chemical Proteomics Reveals Ferrochelatase as a Common Off-Target of Kinase Inhibitors. ACS Chem. Biol. 2016, 11 (5), 1245-1254. https://doi.org/10.1021/acschembio.5b01063.

(45) Wang, T.-H.; Wang, H.-S.; Soong, Y.-K. Paclitaxel-Induced Cell Death. Cancer 2000, 88 (11), 2619 2628. https://doi.org/10.1002/1097-0142(20000601)88:11<2619::AID-CNCR26>3.0.CO;2-J.

(46) Yang, C.-P. H.; Yap, E.-H.; Xiao, H.; Fiser, A.; Horwitz, S. B. 2-(m-Azidobenzoyl)Taxol Binds Differentially to Distinct $\beta$-Tubulin Isotypes. Proc. Natl. Acad. Sci. 2016, 113 (40), 11294-11299. https://doi.org/10.1073/pnas.1613286113.

(47) Comess, K. M.; McLoughlin, S. M.; Oyer, J. A.; Richardson, P. L.; Stöckmann, H.; Vasudevan, A.; Warder, S. E. Emerging Approaches for the Identification of Protein Targets of Small Molecules - A Practitioners' Perspective. J. Med. Chem. 2018, 61 (19), 8504-8535. https://doi.org/10.1021/acs.jmedchem.7b01921.

(48) Helbig, A. O.; Heck, A. J. R.; Slijper, M. Exploring the Membrane Proteome - Challenges and Analytical Strategies. J. Proteomics 2010, 73 (5), 868-878. https://doi.org/https://doi.org/10.1016/j.jprot.2010.01.005.

(49) Cekic, C.; Linden, J. Adenosine A2A Receptors Intrinsically Regulate CD8+ T Cells in the Tumor Microenvironment. Cancer Res. 2014, 74 (24), 7239-7249. https://doi.org/10.1158/0008-5472.CAN-133581.

(50) Muranaka, H.; Momose, T.; Handa, C.; Ozawa, T. Photoaffinity Labeling of the Human A2A Adenosine Receptor and Cross-Link Position Analysis by Mass Spectrometry. ACS Med. Chem. Lett. 2017, 8 (6), 660-665. https://doi.org/10.1021/acsmedchemlett.7b00138.

(51) Yang, X.; Michiels, T. J. M.; de Jong, C.; Soethoudt, M.; Dekker, N.; Gordon, E.; van der Stelt, M.; 
Heitman, L. H.; van der Es, D.; IJzerman, A. P. An Affinity-Based Probe for the Human Adenosine A2A Receptor. J. Med. Chem. 2018, 61 (17), 7892-7901. https://doi.org/10.1021/acs.jmedchem.8b00860.

(52) Zocchi, C.; Ongini, E.; Conti, A.; Monopoli, A.; Negretti, A.; Baraldi, P. G.; Dionisotti, S. The NonXanthine Heterocyclic Compound SCH 58261 Is a New Potent and Selective A2a Adenosine Receptor Antagonist. J. Pharmacol. Exp. Ther. 1996, 276 (2), 398-404.

(53) Kudlacek, O.; Just, H.; Korkhov, V. M.; Vartian, N.; Klinger, M.; Pankevych, H.; Yang, Q.; Nanoff, C.; Freissmuth, M.; Boehm, S. The Human D2 Dopamine Receptor Synergizes with the A2A Adenosine Receptor to Stimulate Adenylyl Cyclase in PC12 Cells. Neuropsychopharmacology 2003, 28 (7), 1317 1327. https://doi.org/10.1038/sj.npp.1300181.

(54) Defossa, E.; Wagner, M. Recent Developments in the Discovery of FFA1 Receptor Agonists as Novel Oral Treatment for Type 2 Diabetes Mellitus. Bioorg. Med. Chem. Lett. 2014, 24 (14), 2991-3000. https://doi.org/https://doi.org/10.1016/j.bmcl.2014.05.019. 